\title{
Taking tea with actin and microtubules
}

M

icrotubule plus ends may control sites of cell polarity and cytokinesis in both animal and yeast cells, but the mechanisms behind this have remained obscure. Now Fred Chang and colleagues at Columbia University, New York, NY, have detected a protein complex in Schizosaccharomyces pombe that links the actin- and microtubulebased cytoskeletal systems. Its existence also suggests that microtubule plus ends polarize cells by targeting cell-polarity factors to the cell surface.

The two proteins that coimmunoprecipitate are the microtubule-associated Tea1 protein and the actin-associated Bud6 protein. Tea1p is constantly deposited at cell tips by the plus ends of micro-

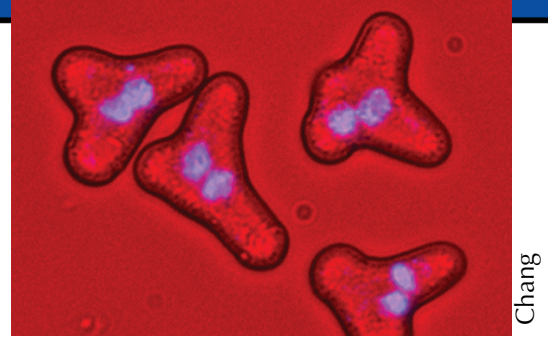

Polarity failure in fission yeast results in T-shaped cells.

tubules. The cell end that has grown before does not need Tea1p to continue growing, but delivery of Tea1p to the new end is apparently necessary for both the initiation of new growth and, according to Chang, the maintenance of Bud6p at this site. The two proteins are found in large multiprotein complexes that may drive the formation of actin-based structures necessary for growth.

Reference: Glynn, J.M., et al. 2001. Curr. Biol. 11:836-845.

\section{Containing the bullet}

A switch from intramolecular to intermolecular disulfide bonds helps Hydra and related jellyfish to construct a capsule of enormous strength, according to a team of zoologists and biochemists.

The capsule, actually a specialized organelle called a nematocyst, is used by Hydra for defense, locomotion, and prey capture. Nematocysts contain a high concentration of

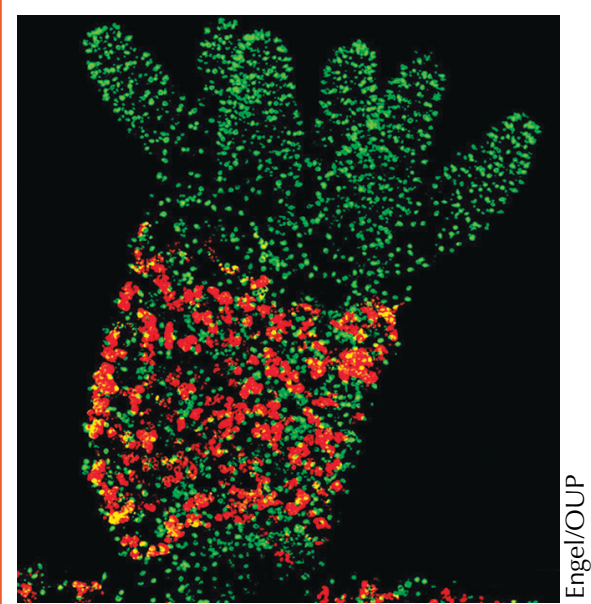

The minicollagen antibody (red) detects nematocysts (green) only in their immature form, before intermolecular disulfides form. poly- $\gamma$-glutamate, resulting in an internal pressure of up to 150 atmospheres that drives the explosive discharge of an internal spiny tubule when the organism is irritated or detects prey. This discharge has a maximum velocity of $2 \mathrm{~m}$ per second and an acceleration of $40,000 \mathrm{~g}$, and is thus one of the fastest cellular processes in nature.

Nematocysts must have a strong wall to contain such pressure. Jürgen Engel (University of Basel, Basel, Switzerland) and colleagues suspected that disulfides might be important, as reducing conditions cause nematocysts to burst. They examined disulfide formation in minicollagen1 , a nematocyst wall component, and found that minicollagen-1 synthesized either recently in vivo, or at any time in a recombinant system, has only intramolecular disulfides. A switch from intramolecular to intermolecular disulfides, detected biochemically and as a reduction in antibody reactivity, was associated with hardening of the nematocyst wall. This process may be similar to the acquisition of intermolecular disulfides by mammalian collagen IV during basement-membrane formation.

Reference: Engel, U., et al. 2001. EMBO J. 12:3063-3073.

\section{Microtubules concentrate}

Two centrosomal proteins help restrict the bulk of microtubule polymerization to the spindle, according to two papers from Fiona Cullen and Hiroyuki Ohkura (University of Edinburgh, Edinburgh, UK) and Jordan Raff (Wellcome/CRC Institute, Cambridge, UK) and colleagues.

The two Drosophila proteins, Minispindles (Msps) and D-TACC, have previously been shown to localize to centrosomes. Here both groups show that the two proteins physically interact. At least in female fly meiotic
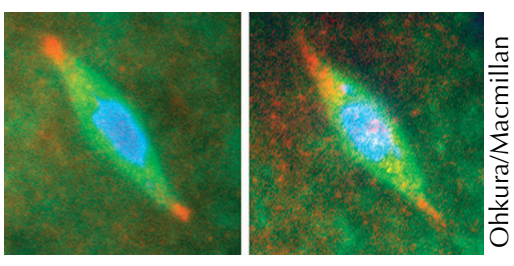

Msps in red localizes to the poles (left) or polar regions (right) of the fly spindles seen in green.

cells, the microtubule motor Ncd helps with the localization of Msps to spindle poles, and this localization is stabilized by the interaction with $D$ TACC at either conventional centrosomal spindle poles (Raff) or the acentrosomal poles that form during female fly meiosis (Cullen and Ohkura). Msps and D-TACC are the first proteins that have been localized to these unusual acentrosomal poles.

Lack of Msps leads to tripolar meiotic spindles, and overexpression of D-TACC (and subsequent recruitment of Msps to aggregates of D-TACC) results in the formation of extra microtubule asters in synctial embryos. Given the fact that Msps promotes microtubule polymerization, both groups suggest that the localization of Msps to spindle poles is essential to focus and restrict microtubule growth to a bipolar spindle.

References: Cullen, C.F., and H. Ohkura. 2001. Nat. Cell Biol. 3:637-642

Lee, M.J., et al. 2001. Nat. Cell Biol. 3:643649. 